THURSDAY, MARCH I8, 1880

\section{DISSOCIATION OF CHLORINE, BROMINE AND IODINE}

I N NATURE, vol. xx. p. 357 , I gave an account of Prof. $I$ V. and Herr C. Meyer's remarkable observations on the density of chlorine at high temperatures, which showed that the chlorine evolved from platinous chloride at temperatures of $\mathrm{s}, 200^{\circ}$ and above had a density only two-thirds of that corresponding to the molecular formula $\mathrm{Cl}_{2}$. I also mentioned that the Meyers had stated that iodine exhibited a similar behaviour.

These observations, tending as they did to show that chlorine was not the simple substance it had hitherto been supposed to be, naturally excited great interest among chemists, and further information has been most anxiously looked for ; it must be admitted, however, that they were received with considerable scepticism, more especially because the statement relating to iodine was in direct contradiction with a most careful series of experiments on the comparative behaviour of air and this substance made by Deville and Troost, who, after assuring themselves that iodine vapour underwent a normal expansion, made use of iodine as a pyrometer in many determinations in the course of their celebrated investigation of the density of a variety of inorganic bodies at furnace temperatures.

This scepticism was considerably strengthened by the appearance, in a recent number of the Comptes Rendus, of a paper by a well-known American chemist, Prof. Crafts, describing a quasi-repetition of the Meyers' experiment with chlorine. The method adopted by Crafts was a slight modification of that introduced by V. Meyer. Two graduated and calibrated U-tubes, maintained at constant temperature by a bath of cold water, were connected with $V$. Meyer's apparatus in such a manner that a known volume of gas could be transferred from the one to the heated bulb of the density apparatus through a very fine tube, the volume of gas displaced by it being collected and measured in the second U-tube. In two experiments made in this manner at the highest temperature of the furnace, the density apparatus being filled with air, 10 c.c. of chlorine displaced 10.37 c.c. and 10.24 c.c. of air; the apparatus being filled with chlorine, Io c.c. of air were found to displace $9^{\circ} 9^{8}$ and ro c.c. of this gas. These experiments were made with a porcelain apparatus ; using a platinum apparatus, to c.c. of chlorine were found to displace $10^{\circ} 43$ c.c. and 10.50 c.c. of air. If the expansion observed by the Meyers had taken place, the quantities of air and of chlorine collected should have been 15 c.c. and 6.6 c.c. respectively, so that operating with free chlorine, Crafts failed to verify the observation of the German chemists.

With iodine, however, he obtained results confirmatory of their statement, the observed density being $60^{\circ}$ r and 593 , instead or 879 , the theoretical number corresponding to the formula $I_{2}$. Bromine was found to be intermediate in its behaviour, the numbers obtained being 4.39 and 4.48 , instead of 5.57 , indicating a reduction in density of one-fifth in place of the reduction of one-third observed in the case of iodine. Hydrogen chloride and carbon dioxide gave VOL. XXI. -No. $54^{2}$ normal results, showing that there was no fault inherent in the method; Crafts, however, noticed that the glaze of the Bayeux porcelain vessels used was much attacked by the coal-gas flame, and that at the high temperatures employed they were slightly porous to hydrogen and water gas, but not to other gases, although not to an extent to vitiate the experiments, only $001-002$ gramme of water passing through in the course of an hour.

The announcement of these results has led Meyer to give an account of experiments he has made in conjunction with Herr Züblin since the publication of the paper by $\mathrm{C}$. Meyer and himself, but prior to the publication of the paper of Crafts. Meyer and Züblin confirm the accuracy of Crafts's observations. Using chlorine gas prepared in the ordinary way, and carefully purified and dried by passing it through water and sulphuric acid and over phosphoric anhydride, they found in three experiments at a yellow heat, $2.57,2.63,2.64$; in mean $2.6 \mathrm{~J}$, instead of $2: 45$, which is the density corresponding to the formula $\mathrm{Cl}_{2}$.

We have then the astonishing result that whereas ready prepared free chlorine is stable at high temperatures, nascent chlorine, i.e., chlorine at the moment of liberation from the compound platinous chloride, is unstable, and undergoes dissociation : for there can now be little doubt that such is the nature of the phenomenon involved in the reduction of its density observed by the Meyers, the argument that this may be due to a great difference in the rate of expansion of chlorine as compared with gases such as oxygen and nitrogen at high temperatures being disposed of by the fact that free chlorine does exhibit a normal behaviour in this respect.

Meyer also publishes the results of a long series of experiments on the density of iodine. In all of these, purified solid iodine was employed and not an iodine compound. The first series of observations, made in a porcelain vessel, are summarised in the following table:-

\begin{tabular}{|c|c|c|c|c|}
\hline Temperature. & & Observed & density. & Theoretical density. \\
\hline 2003 & $8 \cdot 8$ & $8 \cdot 8_{3}$ & & $8 \cdot 78=I_{2}$ \\
\hline About 450 & 8.8 & $8 \cdot 85$ & & \\
\hline $5^{86}$ & $8 \cdot 7$ & $8 \cdot 7 \mathbf{I}$ & $8 \cdot 71$ & \\
\hline,$\quad 842$ & $6 \cdot 68$ & 6.80 & $6 \cdot 80$ & \\
\hline$,, \quad 1,027$ & $5 \%$ & 5.74 & & \\
\hline$, \quad 1,570$ & $5 \cdot 6$ & $5 \cdot 60$ & $5 \cdot 7 \mathrm{I} \quad 5 \cdot 8 \mathrm{I}$ & $5 \cdot 83=\frac{2}{3} I_{2}$ \\
\hline
\end{tabular}

On comparing these results with those for chlorine from platinous chloride, it will be observed that the dissociation of iodine is complete at a considerably lower temperature (about $1,000^{\circ}$ ) than that of chlorine (at about $1,200^{\circ}$ ).

These results being so at variance with those obtained by Deville and Troost at a temperature of $1,040^{\circ}$, Meyer subsequently made further experiments with entirely new apparatus and fresh iodine, but without observing any departure from them. A determination at about $1,052^{\circ}$ in a porcelain apparatus gave 5.88 ; and the density of mercury at the same temperature being simultaneously determined to control this result, the number 6.98 was obtained in place of the theoretical number, 6.91. Two experiments with iodine in a platinum vessel at about I, $567^{\circ}$ gave $5.7 \mathrm{I}$ and $5.8 \mathrm{I}$ as the density. 
The only explanation which can at present be advanced to account for the difference between the observations of Deville and Troost on the one hand, and Meyer and Crafts on the other, is that in the experiments of the former the iodine was gradually converted into vapour, whereas the method adopted by the latter involves the almost instantaneous volatilisation of the iodine; in the case of some organic compounds a difference of this kind in the mode of heating is known to exercise a considerable and in many respects similar influence on the result, so that this explanation is not unsupported by analogy.

Great difficulty was experienced in determining the density of free bromine in consequence of the explosive rapidity with which it is converted into gas when introcluced into the intensely-heated bulb of the density apparatus. The results obtained are not accordant, but all lie between the number corresponding to the molecular formula $\mathrm{Br}_{2}$ and that required on the assumption that dissociation takes place to the same extent as in the case of iodine. Using platinic bromide, $\mathrm{PtBr}_{4}$, however, instead of free bromine, Meyer and Züblin find that a reduction in density takes place precisely of the character of that observed for chlorine from platinous chloride and for iodine. Thus at a temperature of about $1,570^{\circ}$ the observed density in two experiments was 3.78 and 3.64 , 3.64 being exactly two-thirds of the density corresponding to the molecular formula $\mathrm{Br}_{2}$.

As yet Meyer has told us nothing of the nature of the dissociation products of the three halogens; their determination and separation will probably be attended with great experimental difficulties, but the problem could not well be placed in abler hands, and we trust that ere long we may be able to congratulate him on the accomplishment of this the crowning triumph of his labours.

\section{HENRY E. ARMSTRONG}

\section{GLAISHER'S FACTOR TABLES \\ Factor Table for the Fourth Million. By James Glaisher, F.R.S. (London: Taylor and Francis, 1880.)}

THERE is no general method of ascertaining whether one number is divisible, without remainder, by another specified number (less than its half) except by actual trial, or by the knowledge, otherwise acquired, of all the divisors of the first number. If then the second is not among these, it is also known that it is not a divisor of the first number. The knowledge of whether a specified number has any divisors at all, and if so what they are, is only to be obtained in general by trying it with all possible divisors less than its square root. The process can be shortened, but only to a limited extent, and, speaking generally, it would require hundreds of division sums, to ascertain by trial that $3,979,769$ had 1979 for a divisor, and was consequently the product of I 979 and $201 \mathrm{r}$.

It is, however, frequently important to mathematicians to know how to split up any given number into its divisors or factors, and this without the enormous labour which may be involved in actually trying for its divisors, especially as there is no general mathematical principle which enables us to dispense with the trial, or even to shorten it so as to bring it within practicable limits. The alternative is to tabulate numbers up to a given limit, and to indicate, for each, whether it has divisors, and what they are. It is not necessary, or usual, to include in such tables every number without exception; for an inspection of the last figure of any number tells us whether it is divisible by two or by five; and the old rule of "casting out the nines" tells us whether it is divisible by three. These considerations greatly reduce the number which it is necessary to tabulate; for, among the first 300 numbers, 150 are even, that is to say, divisible by 2 ; and of the remaining 150,50 are divisible by 3 ; while of the 100 left after that, 20 are divisible by 5 . The exclusion of the numbers divisible by 2,3 , or 5 thus reduces the number of tabular entries required, from 300 to 80 , and this proportion holds all through the table. as well as for the first 300 numbers. It will be observed that the last two figures of these 80 numbers remain the same for every batch of 300 . This facilitates the tabulation, and advantage has been taken of this facility in printing the Tables.

The first extensive tables of this kind were those published by the Austrian General, Baron von Vega, at the close of the last century. These extended from I to 108,000, and thus give all the divisors of the numbers not divisible by 2,3 , and 5 within those limits. The next table was that of Chernac, a Polish Professor of Mathematics at Deventer, in Over-yssel, which was published in I8II. It contained all the divisors of all numbers, not divisible by 2,3 , and 5 up to I,OI 2,000 . It forms a very thick quarto volume of over $I, \infty 00$ pages.

The next extension was made by Burckhardt (1814-17), who published a series of three volumes, giving, not all the divisors, but the least prime divisor, of all numbers (except those divisible by 2,3 , and 5) up to $3,036,0 \mathrm{co}$. This is not quite so convenient, as a matter of immediate reference, as giving all the divisors; but it answers every necessary purpose. For example, when we know that $3,999,589$ has II for its least divisor, we can find by actual division that the quotient is 363,599 . We "look out" this number in the earlier part of the table, being sure of finding it there, seeing that II was the least divisor of its multiple; we find its least divisor to be $3 x$. Performing the division by $3 \mathrm{I}$, we obtain the quotient I 1,729 . We "look out" this again in the earlier part of the table, and we find that 37 is the least divisor. Performing this division, we obtain 317 as the quotient. Since this is less than $37 \times 37$, we know that it can have no divisors except unity and itself, or that it is prime. If, instead of the least prime divisor, all the divisors had been given, we should at once have found from the table

$$
3,999,589=\text { II } \times 31 \times 37 \times 317 .
$$

There is an obvious advantage in the more complete table. Unfortunately it is balanced by the practical inconvenience of size, and "a great book is a great evil." What this practically comes to may be judged of from the remark that Chernac's table, which gives all the prime factors from I to I, OI 9,000 , takes IO20 quarto pages; while Burckhardt's, which gives only the least prime divisor, contains the numbers from $x$ to $3,036,000$ in 336 quarto pages. It is true that Burckhardt's table is more closely printed than Chernac's, with somewhat smaller type, and a slightly larger form; but, making all allowances, the 